\title{
Waveguide Loss Measurement Using the Reflection Spectrum
}

\author{
W. H. Guo, D. Byrne, Q. Y. Lu, and J. F. Donegan, Senior Member, IEEE
}

\begin{abstract}
A method for waveguide loss measurement purely based on the reflection spectrum is proposed. Using the Fourier series expansion of the single longitudinal mode in the reflection spectrum, the ratio between the second and first harmonics gives the round-trip loss even when the reflection coefficient of the launching optical field directly reflected by the waveguide facet is unknown. The internal loss of a Fabry-Pérot laser diode was measured by the proposed method with results which compare well with those estimated from the amplified spontaneous emission spectrum.
\end{abstract}

Index Terms-Amplified spontaneous emission (ASE), Fourier series expansion, reflection spectrum, waveguide loss.

\section{INTRODUCTION}

$\mathbf{T}$ HE Fabry-Pérot (FP) technique is a convenient and accurate way to measure waveguide losses [1], [2]. It is especially suitable to characterize low loss waveguides because such waveguides generally have clear FP resonances. It is also suitable for measurements of waveguides based on III-V materials since high-quality facets on these waveguides can be easily formed by cleavage. At present the FP method is always based on measuring the transmission spectrum of the FP cavity formed by the waveguide and its two facets. For transmission measurements, access to both facets are needed, which is not available in some cases. For example, in measuring the internal loss of an FP laser diode, the laser always sits on a submount with just one facet accessible. Also the transmission spectrum measurement requires both input and output optical coupling which increases the alignment burden quite a lot. In this letter, we extend the FP method so as to use the reflection spectrum to measure the waveguide loss. The reflection spectrum has been combined with the transmission spectrum to measure the waveguide loss as well as the facet reflectivity [3], [4]. However, as pointed out in [5], these kinds of measurements become problematic if the reflection of the launching optical field deviates from the reflection of the waveguide mode at the waveguide facet, which unfortunately is the general case because the reflection of the launching mode depends sensitively on its mode profile and the incident angle. If assuming that the reflection of the launching mode at the facet is generally different from the reflection of the waveguide mode, the reflection spectrum would become difficult to

Manuscript received April 18, 2008; revised May 26, 2008. This work was supported by SFI under its CSET Centre for Telecommunication Value Driven Research (CTVR), Grant 03/IE3/I405.

The authors are with the Semiconductor Photonics Group, School of Physics and Centre for Telecommunication Value-Chain Driven Research (CTVR), Trinity College, Dublin 2, Ireland (e-mail: guow@tcd.ie).

Digital Object Identifier 10.1109/LPT.2008.927885

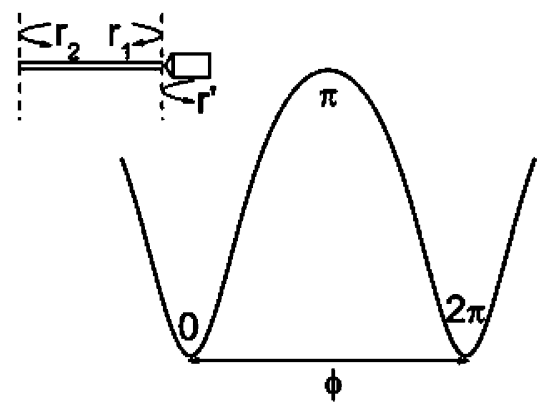

Fig. 1. Schematic diagram of one longitudinal mode of the reflection spectrum. The inset shows schematically a lensed fiber coupled to a waveguide.

use. In this letter, we measured the waveguide loss from just the reflection spectrum based on the Fourier series expansion method [6], [7]. Instead of using the ratio between the first harmonic and the dc term, we use the ratio between the second and the first harmonic terms. As seen from the analysis below, the uncertainty caused by the direct reflection of the launching mode can then be bypassed.

\section{THEORY}

As shown in the inset of Fig. 1, a lensed fiber is used to launch light into the waveguide and also couple the reflection back for monitoring. The reflection spectrum measured will include two parts: one is the direct reflection of the launching mode by the waveguide facet, the other is the light coupled into the waveguide and reflected back through the FP cavity. The total reflection amplitude can be expressed as

$$
\chi=r^{\prime}+\Gamma t_{1}^{2} \frac{b}{r_{1}} \exp (-j \phi) \sum_{p=0}^{\infty}(b \exp (-j \phi))^{p}
$$

where $r^{\prime}$ is the direct reflection coefficient, $\Gamma$ is the coupling coefficient, $t_{1}$ and $r_{1}$ are transmission and reflection coefficients of the front facet as normally incident with the waveguide mode, $b=\exp (-\alpha l) R, R \equiv r_{1} r_{2}$, is the round-trip loss, $r_{2}$ is the reflection coefficient of the rear facet, $\alpha$ is the waveguide loss, $l$ is the waveguide length, $\phi=2 \beta n_{\mathrm{eff}} l$ is the round-trip phase shift, $\beta$ is the wavenumber in vacuum, and $n_{\mathrm{eff}}$ is the effective index of the waveguide. The measured reflection spectrum would be

$$
\begin{aligned}
X=r^{2}+\frac{\Gamma^{2} t_{1}^{4} b^{2}}{r_{1}^{2}\left(1-b^{2}\right)} & \left(1+2 \sum_{p=1}^{\infty} b^{p} \cos (p \phi)\right) \\
& +2 \Gamma r^{\prime} t_{1}^{2} \frac{b}{r_{1}} \sum_{p=0}^{\infty} b^{p} \cos ((p+1) \phi) .
\end{aligned}
$$




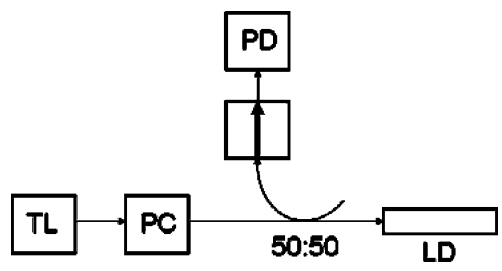

Fig. 2. Schematic diagram of the experiment setup. TL: Tunable laser source. PC: Polarization controller. PD: Photodiode. LD: Laser diode.

As we know from the transmission spectrum, the round-trip loss can be calculated from the modulation depth directly. However, this process can not be applied to the reflection spectrum because of the unknown direct reflection coefficient $r^{\prime}$ which is influenced very much by the launching optical field profile and the incident angle. It could be different from measurement to measurement. Here we introduce a Fourier series expansion process which can bypass this influence. Assuming that we have measured the reflection spectrum which covers at least one longitude mode interval as shown in Fig. 1, we calculate the following Fourier series coefficients [6]:

$$
\tilde{X}_{p}=\frac{1}{2 \pi} \int_{0}^{2 \pi} X(\phi) \exp (-i p \phi) d \phi
$$

From the definition we can find that

$$
\begin{aligned}
& \tilde{X}_{0}=r^{2}+\frac{\Gamma^{2} t_{1}^{4} b^{2}}{r_{1}^{2}\left(1-b^{2}\right)} \\
& \tilde{X}_{1}=\frac{\Gamma^{2} t_{1}^{4} b^{3}}{r_{1}^{2}\left(1-b^{2}\right)}+\Gamma r^{\prime} t_{1}^{2} \frac{b}{r_{1}} \\
& \tilde{X}_{2}=\tilde{X}_{1} b .
\end{aligned}
$$

So we can obtain the round-trip loss from

$$
b=\frac{\tilde{X}_{2}}{\tilde{X}_{1}} .
$$

It is also easy to prove that the above extraction process is not influenced even if there is some external reflection which does not oscillate with wavelength as quickly as the reflection spectrum given in (2). Practical measurement always involves some quantity changing with wavelength which can be easily changed to phase in one longitudinal mode through the relation

$$
\phi=2 \pi \frac{\lambda-\lambda_{0}}{\lambda_{2 \pi}-\lambda_{0}} .
$$

Once we get the round-trip loss we can calculate the loss from $\alpha=-\ln (b / R) / l$, which is the standard process of the FP technique. For cleaved facets of III-V waveguides, $R$ is close to 0.3 . If $R$ is uncertain in practice, two waveguides with different lengths and similar other parameters can be measured simultaneously and loss can be calculated from $\alpha=-\Delta(\ln (b)) / \Delta l$. The coupling conditions will not influence the loss extraction which is the merit of the FP technique.

\section{EXPERIMENT}

The experiment setup used is shown in Fig. 2. The waveguide we measure is basically an InGaAlAs multiquantum-well (QW) FP laser diode. The peak gain wavelength is around $1480 \mathrm{~nm}$ at

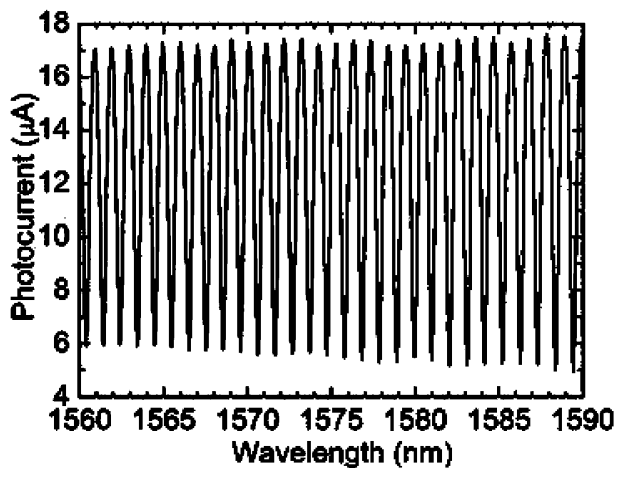

Fig. 3. Reflection spectrum measured from the InGaAlAs FP laser diode.

the laser threshold current. A simple 50:50 $2 \times 2$ beam splitter was used to launch light into the laser diode and also receive the reflection. All the fiber connectors have angled facets to avoid unfavorable reflections. An isolator was placed before the photodiode because the photodiode we use has a relatively small return loss. The polarization controller which consists of a polarizer followed by a quarter-wave plate and a half-wave plate was used to ensure that just the transverse-electric mode of the laser diode is excited. This was realized by treating the laser diode as a photodiode and maximizing the photocurrent by adjusting the wave plates in the polarization controller while launching 1510-nm light into it. A polarization-maintaining antireflection-coated lensed fiber with a spot size $\sim 7 \mu \mathrm{m}$ was used to couple to the laser diode. The coupling was rather easy using forward-biased pumping of the laser diode and monitoring the emission coupled from the lensed fiber. The external tunable laser was scanned with a step of $0.02 \mathrm{~nm}$ from 1560 to $1590 \mathrm{~nm}$ (the longer end of this wavelength range is already deep into the bandgap of the QWs) and a constant power of $1.5 \mathrm{~mW}$. The photocurrent recorded by a Keithley picoameter is shown in Fig. 3. Data with a high signal-to-noise ratio was recorded. During this measurement no current was injected into the laser diode. The loss would be from two parts: one is the internal loss of the laser waveguide caused by scattering from the waveguide sidewalls and intervalence-band absorption in the optical confinement layers, etc; the other part is from the absorption in the QWs. The first part is generally not dependent on wavelength, but the second part is wavelength-sensitive. So the round-trip loss can be expressed as

$$
b=\exp \left(\left(\gamma g(\lambda)-\alpha_{\text {in }}\right) l\right) R
$$

where $\gamma$ is the optical confinement factor, $g(\lambda)$ is the absorption caused by the QWs, and $\alpha_{\text {in }}$ is the internal loss. If the measured wavelength is deep into the QW bandgap, the absorption $g(\lambda)$ would be zero and the round-trip loss becomes $b=\exp \left(-\alpha_{\text {in }} l\right) R$ which as stated above is insensitive to wavelength changes.

By applying the scheme introduced above, the round-trip loss obtained is shown in Fig. 4. As analyzed in [6], using the ratio of the second and first harmonic would be more sensitive to the influence of noise. However, by averaging the results from multiple longitudinal modes, the results can be improved quite 


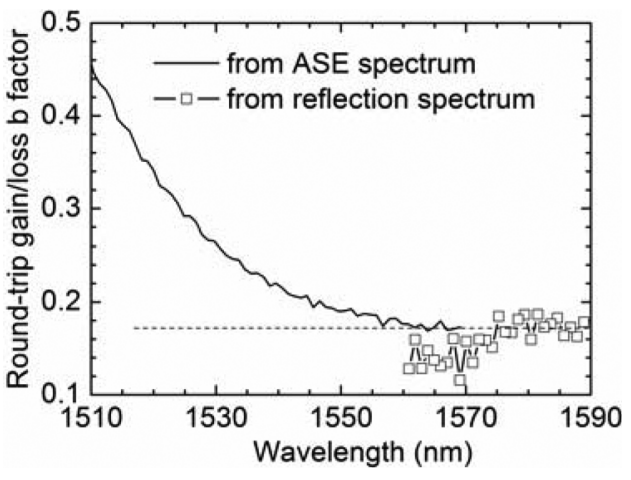

Fig. 4. Round-trip gain measured from the ASE spectrum and the round-trip loss measured from the reflection spectrum. The dashed line indicates the estimated round-trip loss from the ASE measurement.

a lot because the noise influence is reduced when more sampling points are used in the Fourier series expansion process. The averaging is possible because the loss deep into the QW bandgap is a slowly varying function of wavelength. By averaging the round-trip loss in the wavelength range between 1580 and $1590 \mathrm{~nm}$, the value obtained finally was $0.173 \pm 0.009$. With a facet reflection $R=0.3$, the internal loss of the laser diode with a length around $350 \mu \mathrm{m}$ would be $15.8 \pm 1.5 \mathrm{~cm}^{-1}$. To confirm the result we also measured the amplified spontaneous emission (ASE) in the long wavelength part of the ASE spectrum [8]. The current injected into the laser was $12 \mathrm{~mA}$ which is already close to the threshold current. The optical spectrum analyzer resolution bandwidth was set at $0.1 \mathrm{~nm}$. Because there is current injection, the original absorption $g(\lambda)$ in (9) could now become gain as long as the corresponding wavelength is above transparency. However, deep into the QW bandgap there would be nearly no gain so the round-trip gain there is basically the round-trip loss $b=\exp \left(-\alpha_{\mathrm{in}} l\right) R$ [8] (we still call it round-trip gain in this situation as we have current injected). So the results obtained from the ASE spectrum or from the reflection spectrum should match with each other as for wavelength deep into the bandgap. The Fourier expansion method was used to calculate the round-trip gain [6]. The result is also shown in Fig. 4. As seen from it, the round-trip loss estimated from the ASE spectrum measurement agrees well with those derived from the reflection spectrum.

\section{CONCLUSION}

In summary, a method purely based on the reflection spectrum measurement for the waveguide loss extraction is proposed. Measurement was carried out on an FP laser diode which resulted in an internal loss of around $15.8 \mathrm{~cm}^{-1}$ which compares well with the estimation from the ASE spectrum measurement. The use of the second harmonic in the scheme does make the method more sensitive to noise influence. However, generally as loss is not a quickly varying function of wavelength, averaging over several longitudinal modes can be used to generate very reliable results.

\section{ACKNOWLEDGMENT}

The authors would like to thank Eblana Photonics for providing the FP laser diode used in the measurement.

\section{REFERENCES}

[1] R. G. Walker, "Simple and accurate loss measurement technique for semiconductor optical waveguides," Electron. Lett., vol. 21, no. 13, pp. 581-583, Jun. 1985.

[2] R. Regener and W. Sohler, "Loss in low-finesse Ti : $\mathrm{LiNbO}_{3}$ optical waveguide resonators," Appl. Phys. B., vol. 36, no. 3, pp. 143-147, Mar. 1985.

[3] D. F. Clark and M. S. Iqbal, "Simple extension to the Fabry-Pérot technique for accurate measurement of losses in semiconductor waveguides," Opt. Lett., vol. 15, no. 22, pp. 1291-1293, Nov. 1990.

[4] B. K. Kang, Y. H. Park, S. Lee, S. S. Choi, J. Lee, T. Kamiya, and S. H. Park, "Measurement of facet reflectivity of antireflection-coated electroabsorption modulator using induced photocurrent," IEEE Photon. Technol. Lett., vol. 13, no. 2, pp. 112-114, Feb. 2001

[5] W. J. Tomlinson and R. J. Deri, "Analysis of a proposed extension to the Fabry-Pérot technique for measurements of losses in semiconductor optical waveguides," Opt. Lett., vol. 16, no. 21, pp. 1659-1661, Nov. 1991.

[6] W. H. Guo, Q. Y. Lu, Y. Z. Huang, and L. J. Yu, "Fourier series expansion method for gain measurement from amplified spontaneous emission spectra of Fabry-Pérot semiconductor lasers," IEEE J. Quantum Electron., vol. 40, no. 2, pp. 123-129, Feb. 2004.

[7] D. Byrne, W. H. Guo, R. Phelan, Q. Y. Lu, J. F. Donegan, and B. Corbett, "Measurement of linewidth enhancement factors for InGaAlAs laser diode by Fourier series expansion method," Electron. Lett., vol. 43, no. 21, pp. 1145-1146, Oct. 2007.

[8] L. J. P. Ketelsen, "Simple technique for measuring cavity loss in semiconductor lasers," Electron. Lett., vol. 30, no. 17, pp. 1422-1424, Aug. 1994. 